\title{
Vehicle Counting and Moving Direction Identification Based on Small-Aperture Microphone Array
}

\author{
Xingshui Zu ${ }^{1,2}$, Shaojie Zhang ${ }^{3}$, Feng Guo ${ }^{1,2}$, Qin Zhao ${ }^{1,2}$, Xin Zhang ${ }^{4}$, Xing You ${ }^{1}$, \\ Huawei Liu ${ }^{1}$, Baoqing $\mathrm{Li}^{1}{ }^{1 *}$ and Xiaobing Yuan ${ }^{1}$ \\ 1 Science and Technology on Microsystem Laboratory, Shanghai Institute of Microsystem and \\ Information Technology, Chinese Academy of Sciences, Shanghai 201800, China; zuxs@mail.sim.ac.cn (X.Z.); \\ GuoFeng@mail.sim.ac.cn (F.G.); qinzhao21@mail.sim.ac.cn (Q.Z.); livia138567@126.com (X.Y.); \\ liuhw@mail.sim.ac.cn (H.L.); sinowsn@mail.sim.ac.cn (X.Y.) \\ 2 University of Chinese Academy of Sciences, Beijing 100049, China \\ 3 School of Mechanical Engineering and Electronic Information, China University of Geosciences, \\ Wuhan 430074, China; 20141001117@cug.edu.cn \\ 4 IBM-Research China Labotatory, Beijing 100094, China; zxinscholar@gmail.com \\ * Correspondence: sinoiot@mail.sim.ac.cn; Tel.: +86-186-2194-5060
}

Academic Editors: Xiaoning Jiang and Chao Zhang

Received: 9 March 2017; Accepted: 9 May 2017; Published: 10 May 2017

\begin{abstract}
The varying trend of a moving vehicle's angles provides much important intelligence for an unattended ground sensor (UGS) monitoring system. The present study investigates the capabilities of a small-aperture microphone array (SAMA) based system to identify the number and moving direction of vehicles travelling on a previously established route. In this paper, a SAMA-based acoustic monitoring system, including the system hardware architecture and algorithm mechanism, is designed as a single node sensor for the application of UGS. The algorithm is built on the varying trend of a vehicle's bearing angles around the closest point of approach (CPA). We demonstrate the effectiveness of our proposed method with our designed SAMA-based monitoring system in various experimental sites. The experimental results in harsh conditions validate the usefulness of our proposed UGS monitoring system.
\end{abstract}

Keywords: vehicle counting; moving direction; small-aperture microphone array; UGS

\section{Introduction}

The motion parameters of vehicles are important intelligence for a unattended ground sensor (UGS) system and Intelligent Transport System (ITS) [1-6]. The fixed-point observation technique is widely employed in UGS and ITS by installing an inductive loop sensor [7], ultrasonic sensor [8,9], seismic sensor [10] and camera [11]. However, these sensing systems suffer from complicated installation, expensive maintenance costs and high power consumption.

The microphone array (MA) sensor provides low cost, low power consumption, and non-line-of-sight measurement, which is widely used for acquiring military intelligence of intruding targets [1,3,6,12]. A network of MA-based surveillance sensors remotely deployed in conjunction with a command center can provide early warning and assessment of enemy threats, near real-time situational awareness to commanders, and may reduce potential hazards to soldiers [13]. Furthermore, the employment of small-aperture microphone array (SAMA) as a monitoring sensor should be an undetectable system which can avoid visual problems and be cheaper than most currently used systems.

The MA-based detection and classification of moving targets in UGS have received much attention [14-18]. However, relatively less attention has been paid to the counting and moving 
direction estimation in UGS. This issue generally relates to the detection of moving targets, and more specifically to the identification of the number and moving direction of vehicles using one or more SAMA sensors. More particularly, this issue pertains to a system that uses acoustic sensors to acquire enough intelligence on moving targets in dynamic, noisy, and highly mobile environments. An 'acoustic trip line' counter was introduced in $[6,13]$ to detect the harmonic components in the prescribed look-direction by MA with the radius of $0.612 \mathrm{~m}$. Moreover, the method of collaborative signal processing of a pair of $1 \mathrm{~m}$ spacing MA sensors was designed in [19] to determine the number of targets. Nevertheless, those currently used large aperture systems destroyed the stealth and installability characteristic of acoustic sensor in UGS. On the other hand, traffic monitoring systems with MA applied in ITS are proposed in [1-3,20]. However, both of the methods proposed in [1,2] need channel synchronization and ultra-high sampling frequency $(48 \mathrm{kHz})$, which are not realistic in a UGS system. Furthermore, a system based on the amplitude or energy of vehicle-generated sound is easily spoofed or counter measured in military application.

The counting of vehicles and the estimation of their moving direction with small aperture and lower sampling rate are a knotty problem. Another challenge for the SAMA sensor in the wild environment is the wind-generated noise. The sound of the vehicle in the real-world environment has free-field characteristics, and the wind noise has noise-field characteristics [21]. It is well known that the wind noise is unavoidable since it cannot be totally removed by a wind shield. According to [21], spatial coherence could be used to distinguish between the noise of the wind and the sound of the vehicle for each frequency bin. Therefore, we employed a spatial coherence-based method to select the useful bands for determining the vehicle direction, counting vehicles and estimating their moving direction.

In this paper, a SAMA-based system for counting vehicles and estimating their moving direction is provided and its aperture is only $4 \mathrm{~cm}$. We defined a decision zone (DZ) near to the closest point of approach (CPA) to identify the number and moving direction of vehicles. Since the direction of arrival (DOA) estimation error around the CPA is relatively small, we can achieve higher estimation accuracy. The interference of wind noise in the real-world environment is reduced through the estimation of the useful frequency bands by spatial coherence.

This paper is organized as follows. Section 2 illustrates the design of the SAMA sensor system, including the system hardware architecture and the DOA estimation algorithm. Section 3 describes the vehicle counting and moving direction estimation scheme based on the calculated DOA. System verification and experimental results in different situations are given in Section 4 and conclusions are presented in Section 5.

\section{Design of the Estimation System}

\subsection{SAMA System Architecture Design}

In general, the uniform array can provide balanced space for circuit design and the uniform circular array (UCA) has the same resolution in all directions. The vehicle signal occupies the frequency bands from $100 \mathrm{~Hz}$ to $3000 \mathrm{~Hz}$ [22]. The aperture of the array has to satisfy the spatial sampling criterion in all the frequency bands to avoid performance degradation due to spatial aliasing. Therefore, to satisfy the spatial sampling criterion $d \leq 0.5 \lambda$, the array aperture should be no bigger than $5 \mathrm{~cm}$, where $\mathrm{d}$ is the minimum distance between any two array microphones, and $\lambda$ is the wavelength of the acoustic signal. Finally, uniform circular geometry with an aperture of $4 \mathrm{~cm}$ is employed to deploy the microphone [23].

The block diagram of the prototype SAMA system is depicted in Figure 1. The system is divided into three modules according to their functions: MA module (Module 1: MA); preprocessing and sampling module (Module 2: P\&S); and real-time processing or data acquisition module (Module 3: P/A). The acoustic signals from the MA module are sampled in the P\&S module to obtain four simultaneous digital signals. The synchronized filters and amplifiers mean that a comparatively strict 
demand on the consistency of the four channels is requested. The function of the P/A module is configured by users, either for real-time processing by digital signal processing (DSP) or for storing the signals in the memory device for further analysis.

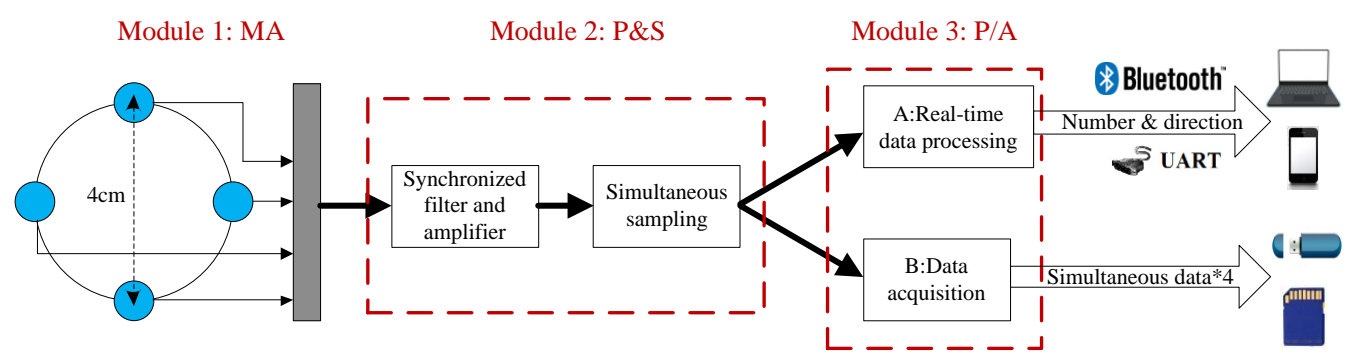

Figure 1. Block diagram of the small-aperture microphone array (SAMA) system hardware architecture.

As shown in Figure 2, the system consists of a mainboard as well as an extended board connecting by a flexible printed circuit. The mainboard consists of a UCA system with four ADMP504 MEMS microphones (Analog Devices, Norwood, MA, USA), a DSP (ADSP21375, Analog Devices, Norwood, MA, USA) as the core processor, MAXIM MAX11043, four-Channel, 16-Bit analog-to-digital converters (ADCs) (Maxim Integrated Products, Sunnyvale, CA, USA) and supplemental hardware circuits. The MAX11043 contains one versatile filter block and programmable-gain amplifier per channel. The extended board contains a CSR BC6415 Bluetooth module (Cambridge Silicon Radio, Cambridge, UK), a data acquisition interface and debug interface. The hardware components that make up the system are illustrated in Figure 2. In general, the aperture of our system is very small $(4 \mathrm{~cm})$ which is an advantage for portability and mobility, but a challenge for high accuracy DOA estimation [23].

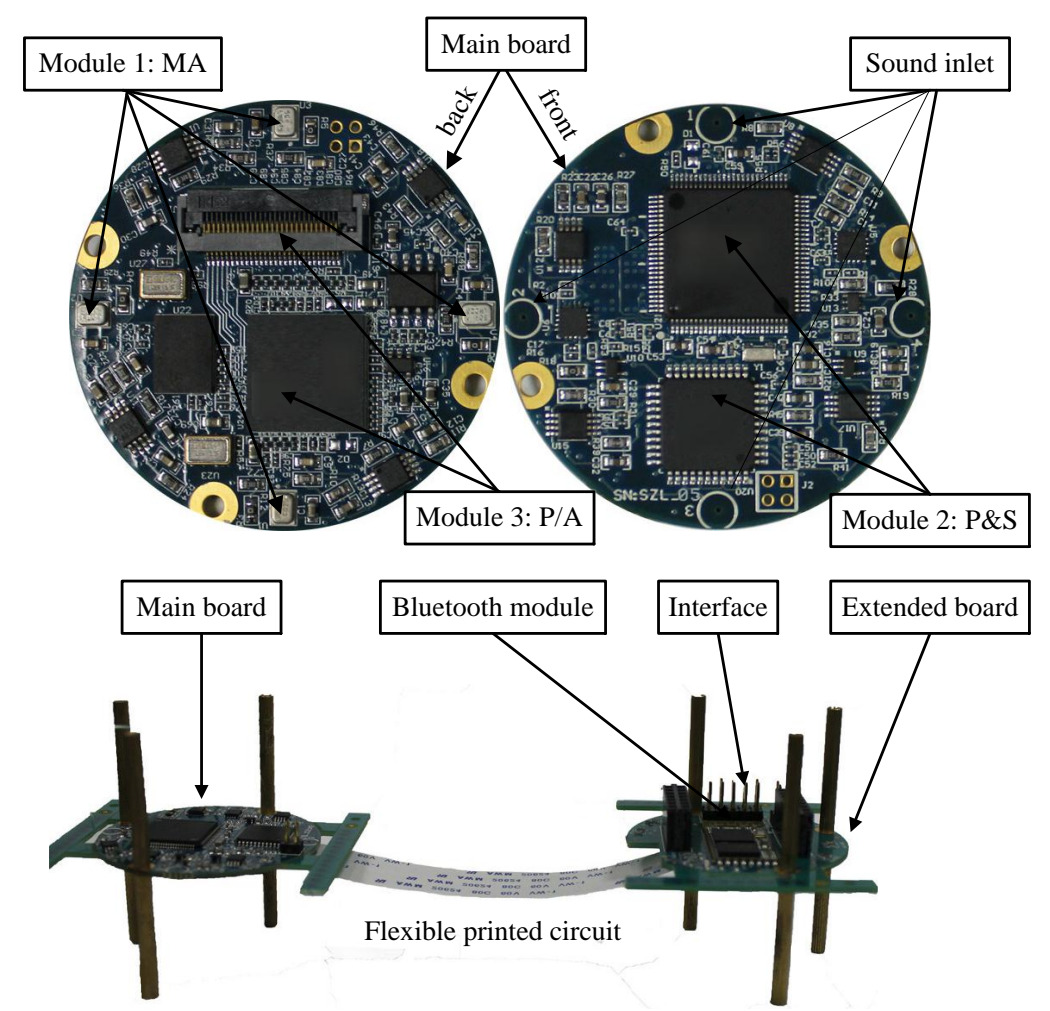

Figure 2. Photograph of the SAMA system; array aperture is $4 \mathrm{~cm}$. 


\subsection{DOA Estimation with Spatial Coherence}

DOA estimation using acoustic signals is inevitably contaminated by wind noise which is the most common interference in an outdoors environment. The wind turbulence on the microphone is comparatively incoherent, and its speed is much slower than that of sound [24]. Spatial coherence is a similarity indicator for signals in the frequency domain. It describes the coherence between two measures at two locations [21]. The spatial coherence function between two microphone signals, $x_{1}$ and $x_{2}$, is equal to the cross power spectrum $G_{x_{1} x_{2}}(f)$ divided by the square root of the product of the two auto-power spectra. Specifically, the spatial coherence of $x_{1}$ and $x_{2}$ is defined by Equation (1):

$$
\gamma_{x_{1} x_{2}}(f)=\frac{G_{x_{1} x_{2}}(f)}{\sqrt{G_{x_{1} x_{1}}(f) G_{x_{2} x_{2}}(f)}}
$$

where $f$ denotes the frequency of interest. The complex cross power spectrum defined in Equation (2) is the Fourier transform of the cross correlation of $x_{1}$ and $x_{2}$ in Equation (3).

$$
\begin{gathered}
G_{x_{1} x_{2}}(f)=\int_{-\infty}^{\infty} R_{x_{1} x_{2}}(\tau) e^{j 2 \pi f \tau} d \tau, \\
R_{x_{1} x_{2}}(\tau)=E\left[x_{1}(t) x_{2}(t+\tau)\right] .
\end{gathered}
$$

Here, $x_{1}$ and $x_{2}$ are two different channel signals from SAMA and $E$ denotes the mathematical expectation (for ergodic random processes the ensemble average can be replaced by a time average). Carter [25] gives an analytical estimation of the bias $E\left[\left|\hat{\gamma}_{x_{1} x_{2}}\right|^{2}\right]-\left|\hat{\gamma}_{x_{1} x_{2}}\right|^{2}$ as a function of the true spatial coherence $\left|\hat{\gamma}_{x_{1} x_{2}}\right|^{2}$, the fast fourier transformation (FFT) time duration $T$ and the time delay $D$.

$$
E\left[\left|\hat{\gamma}_{x_{1} x_{2}}\right|^{2}\right]-\left|\hat{\gamma}_{x_{1} x_{2}}\right|^{2} \cong \frac{-2|D|}{T}\left|\hat{\gamma}_{x_{1} x_{2}}\right|^{2}+\left(\frac{|D|}{T}\right)^{2}\left|\hat{\gamma}_{x_{1} x_{2}}\right|^{2}
$$

In our case, $T=125 \mathrm{~ms}$ (1024 sampling points), $D=8.31 \times 10^{-5} \mathrm{~s}$ (array aperture of $4 \mathrm{~cm}$ ). Figure 3a shows the acoustic signal of a car passing the SAMA sensor and the wind scale [26] is 4 . Spatial coherence is depicted in Figure $3 b$ to show whether the frequency bin is dominated by vehicle or wind noise. To identify the useful frequency band of the signal, we check whether the spatial coherence is above the threshold in each frequency bin. In this paper, 0.7 is chosen by simulation and experiment. If the spatial coherence of a certain frequency bin is larger than 0.7 , then this bin will be selected for direction finding and other uses; otherwise, it will be discarded.

An improved multiple signal classification (MUSIC) algorithm is employed to DOA estimation associated with spatial coherence to discriminate between the wind noise and the acoustic signal of a vehicle. The algorithm first tests the spatial coherence for each frequency bin, then identifies the useful frequency bands for wind noise robust DOA estimation. Details of identifying spatial coherence and selecting useful frequency bands for DOA estimation are discussed in [23].

In addition, inspired by [2], we designed an inaccurate angle regulation (IAR) method to adjust the estimated target angle. One angle value calculation is performed on a signal length of $125 \mathrm{~ms}$ (frame length) and the frame moving step is equal to the frame length. If motion speed is $60 \mathrm{~km} / \mathrm{h}$, the vehicle moves about $2 \mathrm{~m}$ at this time interval, which corresponds to the angle deviation of $0^{\circ}-11^{\circ}$ (assuming that sensors are displaced $10 \mathrm{~m}$ away from the road). This angle deviation depends upon the location of the vehicle in relation to the MA. We conclude that it is not the correct angle of the vehicle and that maybe it is falsely estimated if the two closest neighbor angle values differ more than a deviation of $11^{\circ}$ (deviation threshold). Then, the neighboring angles will linear fitting out a value to replace it. Following the aforementioned principle, the deviation threshold is proportional to the speed of the vehicle. 


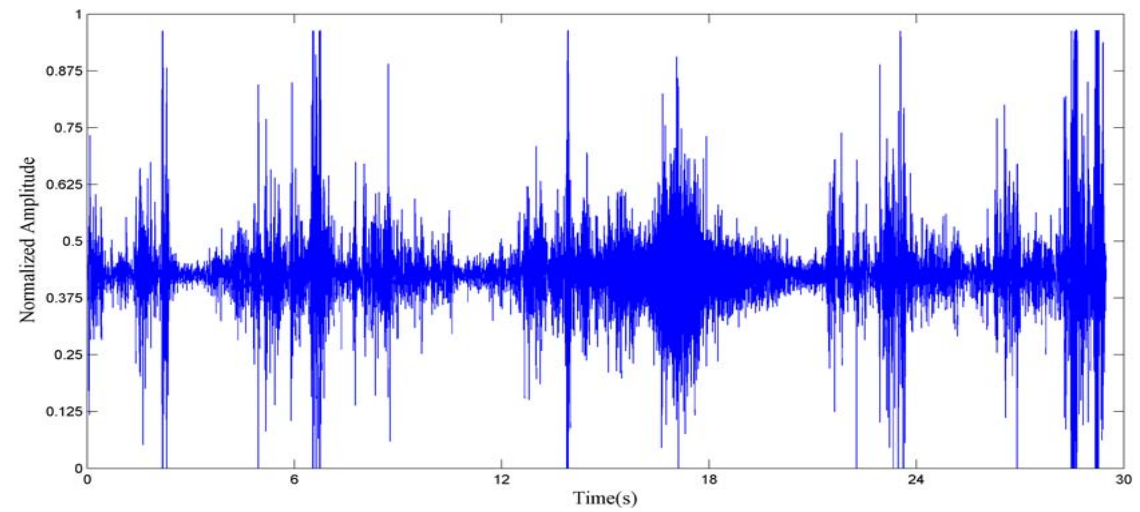

(a)

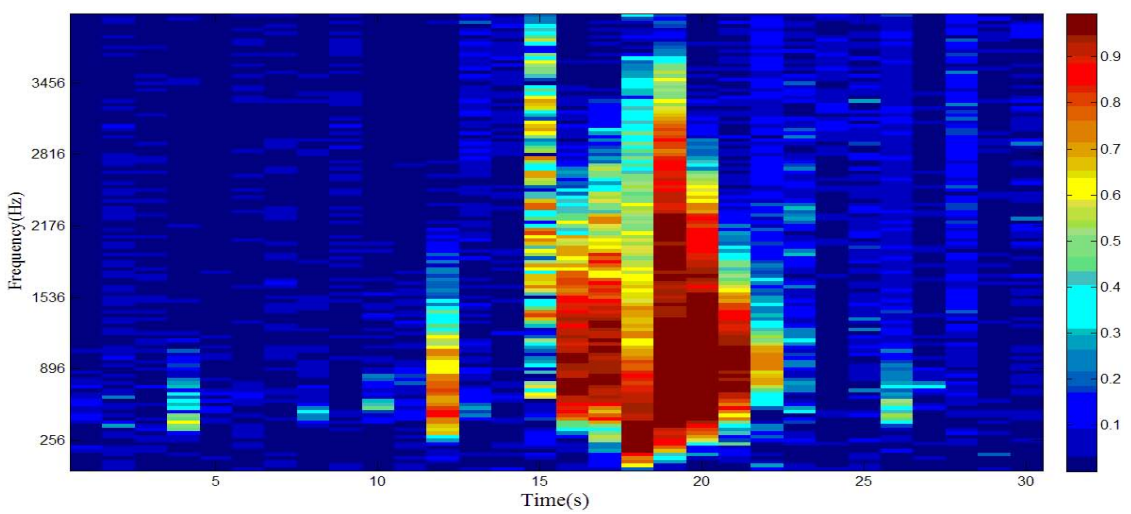

(b)

Figure 3. (a) Acoustic signal of a car passing the SAMA in an outfield test; (b) Spatial coherence of (a).

\section{Vehicle Counting and Moving Direction Estimation}

In this section, we describe the number and moving direction estimation method of vehicles travelling on a previously established route with the calculated DOA. Through statistical analysis, the varying trend of vehicle's bearing angles can provide the number information of vehicles, as shown in Figure 4a. Since the angle estimation error around the CPA is relatively small, we defined a DZ $\left(-15^{\circ},+15^{\circ}\right)$ around the CPA $\left(0^{\circ}\right)$ for subsequent processing. Then, we designed a vehicle counting and moving direction estimation method by analyzing the relationship of the angles with the DZ as described in Figure 5. The reference coordinate system is shown in Figure 6b.

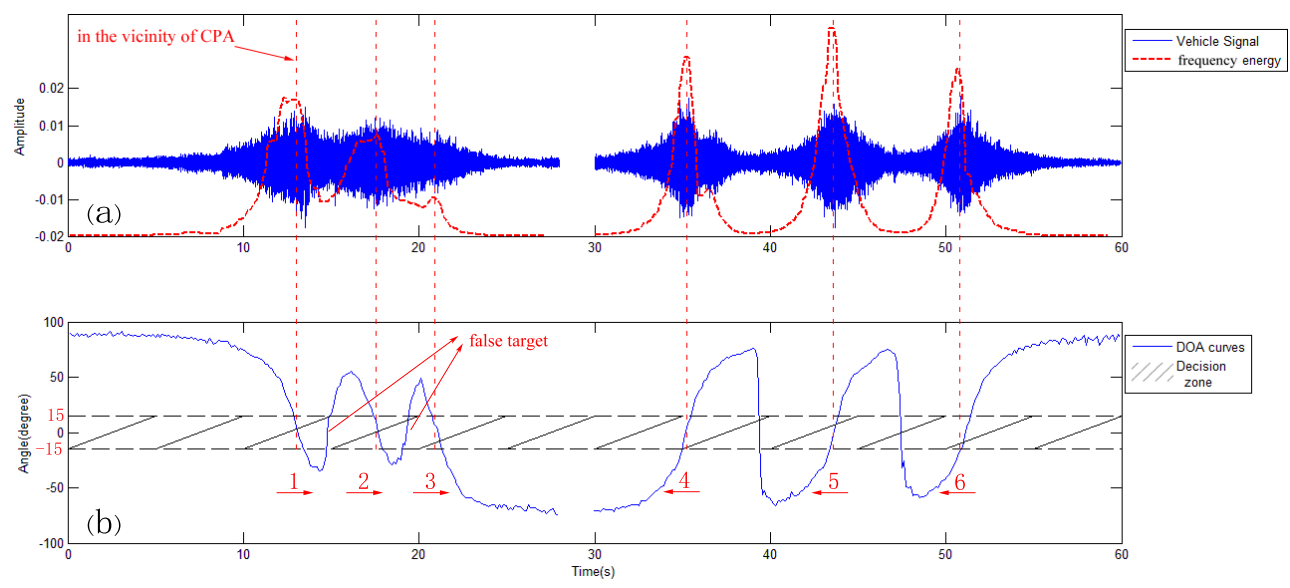

Figure 4. (a) Six vehicles passing through SAMA sensors within $1 \mathrm{~min}$; (b) the corresponding direction of arrival (DOA) curves of (a). 


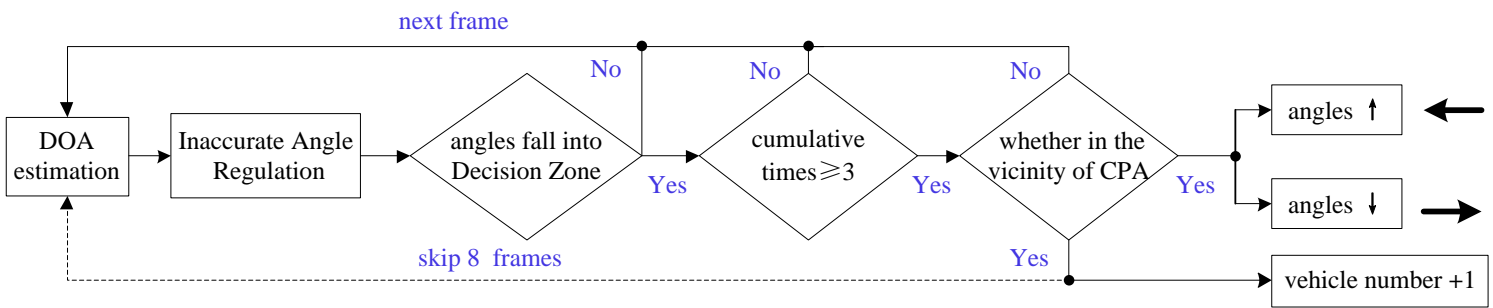

Figure 5. The flowchart of the algorithm for vehicle counting and moving direction estimation.

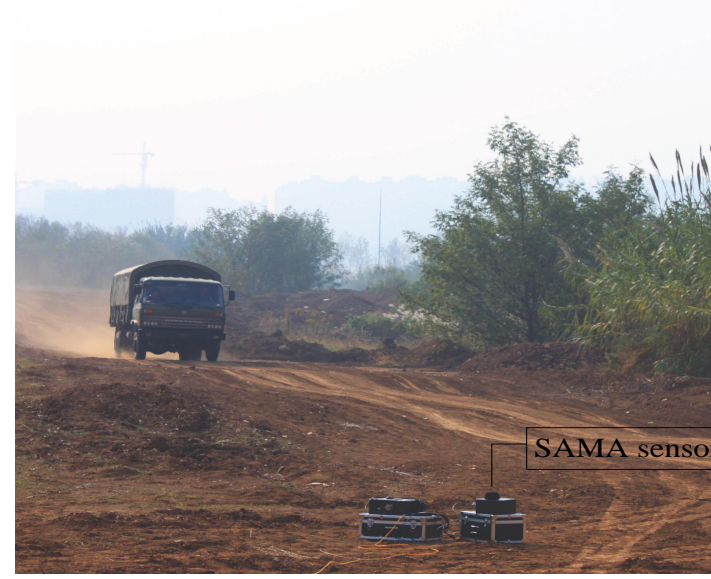

(a)

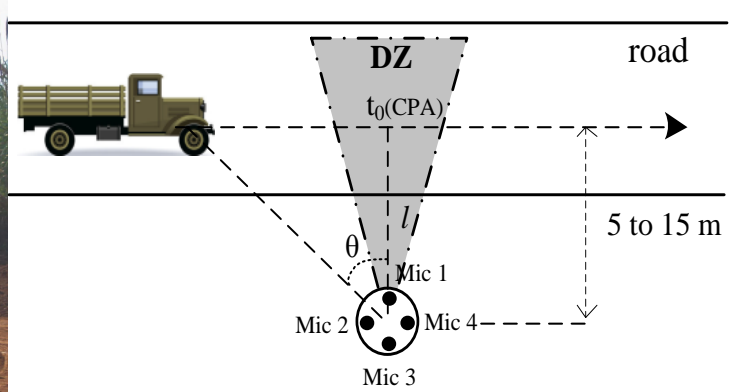

(b)

Figure 6. (a) Photograph of the recording setup; (b) Illustration of the DOA estimation scenario.

Angles that fall into the DZ more than or equal to three times represent a vehicle that was detected passing through a SAMA sensor. Subsequently, the number of vehicles is increased by 1 and the moving direction can be obtained by checking the varying trend of the angles. The vehicle is approaching from the left direction if the angles are gradually decreasing, and conversely from the right direction. At this stage, the detection of a vehicle and its moving direction is finished. After those operations, we skip eight frames to avoid the repeated count of the same vehicle because, in such a short time, another vehicle will not appear.

Assuming that the velocity of the vehicle (denoted as $v$ ) is uniform, then its DOA satisfies the inverse tangent law as Equation (5):

$$
\theta=\arctan \frac{v \cdot\left(t-t_{0}\right)}{l}, t \in R
$$

where $t_{0}$ represents the moment of CPA and $l$ represents the distance between the sensors and lane center, as depicted in Figure 6. Moreover, the ideal DOA curve of three vehicles, without them mutually interference with each other, is shown in Figure 7. However, due to the mutual interference of vehicles, the actual DOA curve of three vehicles passing a SAMA sensor is shown as $0 \mathrm{~s}$ to $30 \mathrm{~s}$ in Figure $4 \mathrm{~b}$. Hence, our method will cause false targets in the middle of two vehicles, as annotated in Figure $4 \mathrm{~b}$. As the DZ is very close to $0^{\circ}$, the vehicle is close to the CPA. Therefore, as shown by the red dotted line in Figure 4a, the frequency energy of selected frequency bands in Section 2.2 is employed to roughly judge whether those frames are in the vicinity of the CPA. Then, we can exclude false targets in order to achieve a highly accurate vehicle count. 


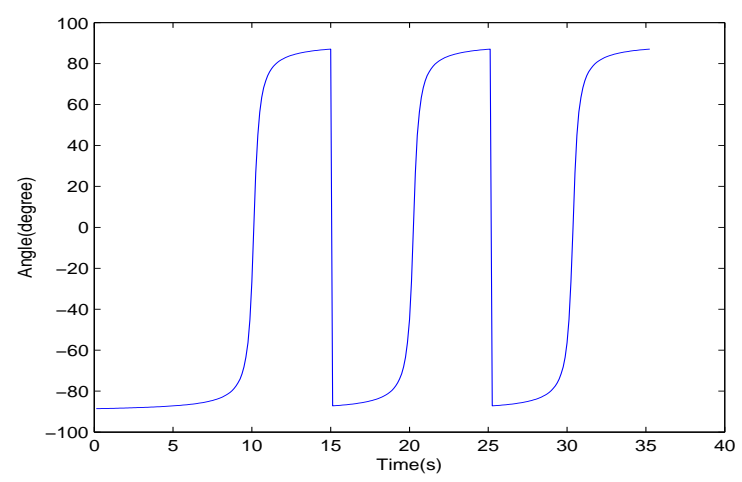

Figure 7. The simulated ideal DOA curve of three vehicles passing a SAMA sensor.

Since counting and moving direction estimation are based on the same vehicle, we should not analyze either of them individually. The estimation of moving direction is executed immediately once a vehicle is counted.

DOA estimation is easily disturbed by other interference signals when the target is far away from the CPA, and the DOA estimation error is positively related to the distance from the CPA. The proposed method, based on the DZ, tightly surrounds the CPA which makes the method more effective.

\section{System Verification and Experimental Results}

\subsection{Experimental Conditions and Datasets}

Ground vehicles are moving targets of focal interest to UGS. Therefore, these three types of vehicles are employed in our experiment and part of their specifications is listed in Table 1. The acoustic signal of a moving target is sampled by the SAMA sensor with a $8192 \mathrm{~Hz}$ sampling rate. Besides, each vehicle is equipped with GPS to obtain the velocity and distance information between the moving target and the sensor. The sensor was located parallel to the road, about 5 to $15 \mathrm{~m}$ away from the lane center and the experimental layout is shown in Figure 6. Meanwhile, wind scales were recorded by an ultrasonic anemometer at the same site during our outfield experiments.

Table 1. Different vehicles' specifications.

\begin{tabular}{cccc}
\hline & \multicolumn{3}{c}{ Vehicle Types } \\
\cline { 2 - 4 } & Car & Truck & Tracked Vehicle \\
\hline Weight $(\mathrm{kg})$ & 1425 & 6800 & 40,200 \\
Number of cylinders & 4 & 6 & 10 \\
Engine capacity & 78 & 170 & 3240 \\
Samples (min) & 107 & 104 & 95 \\
\hline
\end{tabular}

The noise emitted by a vehicle at low and medium speeds is composed of tyre/road noise and mechanically originated noise [27]. Considering the effect of road type and speed, experiments were conducted in different terrains at different speeds [28]. Experimental studies were performed from June 2013 to December 2016 on Chongming Island, Zhoushan Island, Nanjing, Anhui and a suburban district around Shanghai where the wind scales are usually less than 6 . The compositions of our sample set are shown in Table 2 and the photographs of four experimental environments are shown in Figure 8. In those experimental sites, vehicles move at the predetermined velocity of 30 to $60 \mathrm{~km} / \mathrm{h}$ when datasets are collected. Some of the measurement sites are military training grounds with some background activity at times. Samples with higher speeds are not available for the bad road conditions in the real-world environment. Moreover, for the sake of security, experiments of tracked vehicles driving at $60 \mathrm{~km} / \mathrm{h}$ on the concrete road were not implemented. 
Table 2. Experimental datasets recorded in four different experimental sites; every sample is $60 \mathrm{~s}$ with a sampling rate of $8192 \mathrm{~Hz}$.

\begin{tabular}{cccccc}
\hline Recording Location & Chongming & Zhoushan & Fengxian & Nanjing \\
\hline \multicolumn{2}{c}{ Road Type } & Dirt & Concrete & Sand & Mud \\
\hline \multirow{2}{*}{ Vehicle Type Car } & 24 & 30 & 25 & 28 \\
& Truck & 26 & 28 & 22 & 28 \\
& Tracked & 31 & 0 & 30 & 34 \\
\hline \multicolumn{2}{c}{ Total Samples (min) } & 81 & 58 & 77 & 90 \\
\hline
\end{tabular}

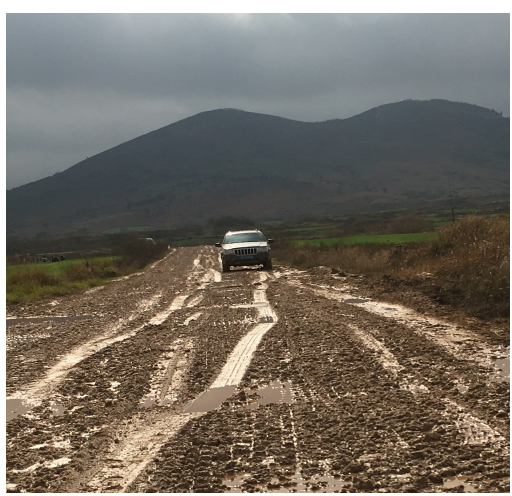

(a)

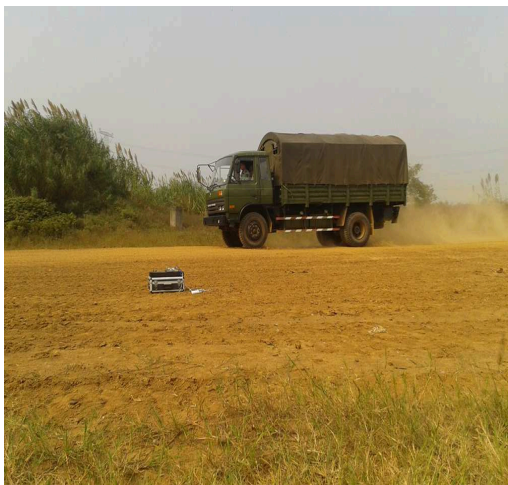

(c)

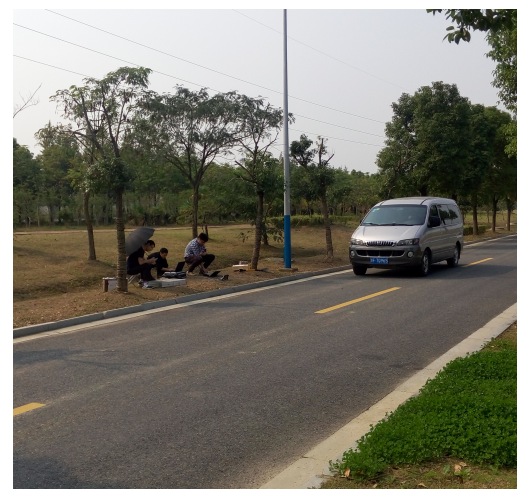

(b)

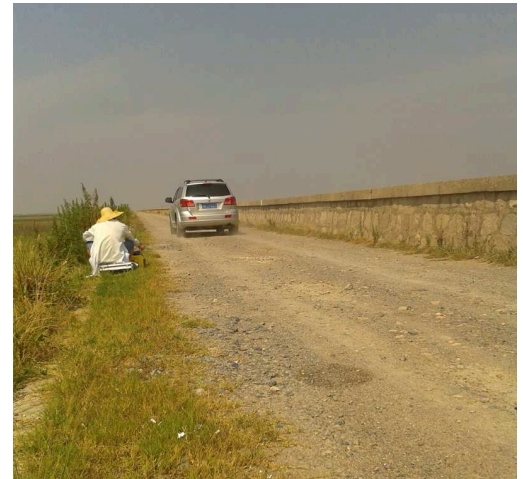

(d)

Figure 8. Four different experimental environments in Nanjing, Anhui and Shanghai. (a) dirt road; (b) concrete road; (c) mud road; (d) sand road.

\subsection{Results and Discussion}

In this section, the experimental data are cropped and a total of 306 min useful acoustic samples from different sites are analyzed. Figure 9 presents the counting accuracy for three types of vehicles in four terrains with different speeds. According to Figure 9, the influence of road type to counting accuracy is greater than that of vehicle speed. In addition, these two factors have the greatest impact on the counting accuracy of cars, while they have less and the least impact on trucks and tracked vehicles, respectively. The faster the speed, the greater the vehicle noise, thus higher counting accuracy can be achieved. Moreover, regardless of the type of vehicle, the counting accuracy on the sand road is the highest among all the tested terrains. 


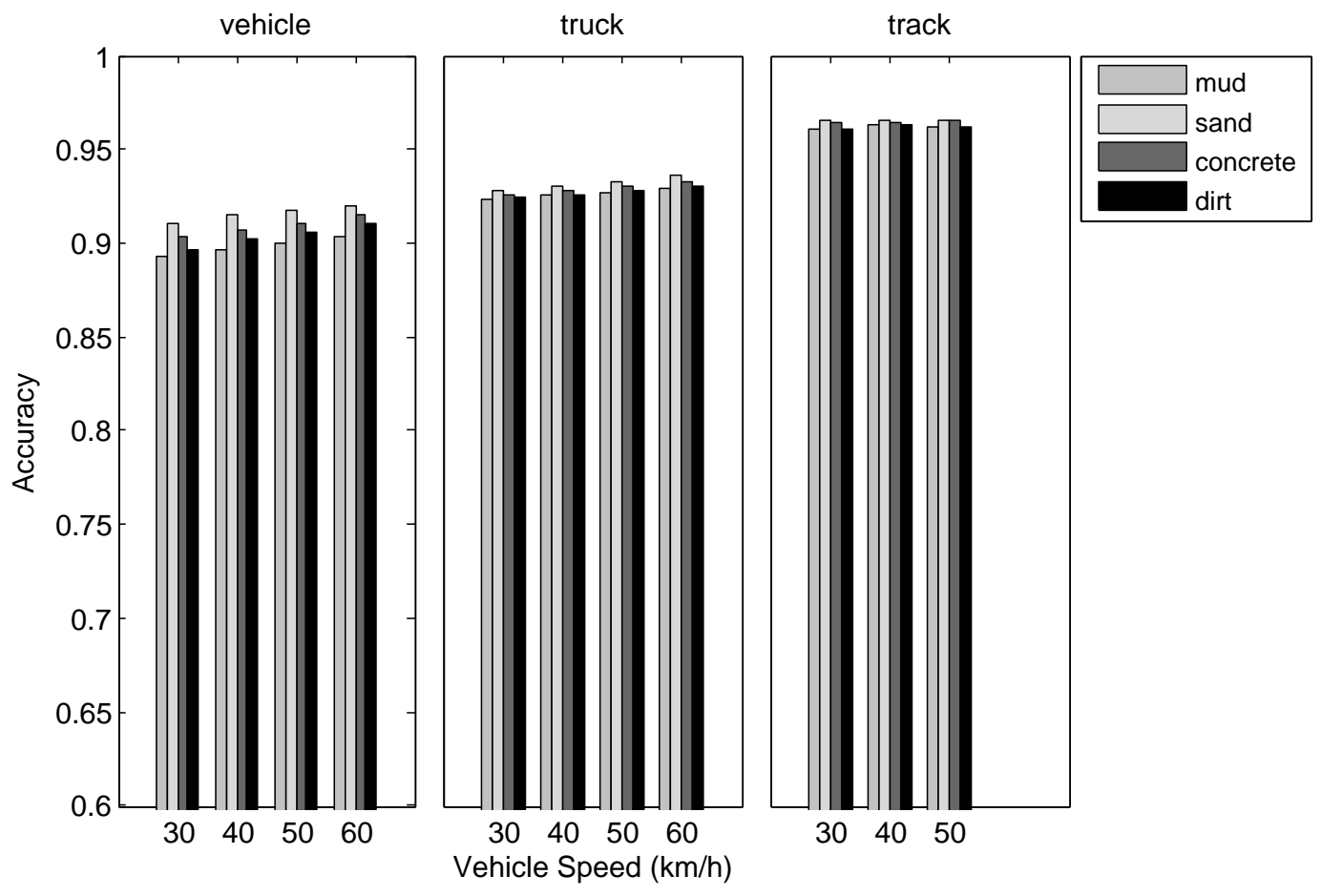

Figure 9. Evaluation results of the vehicle counting algorithm using collected datasets.

The average counting accuracy of three types of vehicles is shown in Table 3 without considering the effect of the terrain and speed. The accuracy of the tracked vehicle is as high as $96.42 \%$ due to its high level of noise. The special counting mechanism ensures that it will not lead to false positive detection, even in strong wind weather conditions. However, ultra-close-distance (less than $20 \mathrm{~m}$ ) driving and overtaking are the main factors that result in false detection. Fortunately, both aforementioned situations, commonly encountered in ITS, are rare in the process of troops moving. Therefore, the proposed method is suitable for monitoring military activity with UGS.

Since counting and moving direction estimation are based on the same vehicle, a one-to-one relationship exists between them. Through our statistics, the moving directions of all correctly counted vehicles are correctly estimated (accuracy: 100\%). It is worth emphasizing that the falsely counted vehicles are not involved in the calculation. Considering the decision mechanism, judging the increasing or decreasing trend of angles in the vicinity of the CPA, the result is reliable. Because of the $100 \%$ estimation accuracy of the moving direction, the overall performance of the system is dominated by counting accuracy.

Considering the application in the field environment, in contrast to ITS, vehicle speed is slow and vehicle flow is limited. Therefore, we can achieve satisfactory results of vehicle counting and moving direction estimation with small aperture and lower sampling rate. However, the ITS has the characteristics of faster vehicle speed, traffic intensity and multi-lane interference. Consequently, it needs large aperture arrays, synchronous acquisition and a very high sampling rate. Those demands lead to a high power consumption and make the UGS system difficult to implement. Hence, we did not compare the performance of the proposed method with methods in [1,2]. Certainly, we should admit that our proposed method shows poor ability in some of the ITS application scenarios.

Table 3. The average accuracy of the counting algorithm, ignoring the effect of the terrain and speeds.

\begin{tabular}{cccc}
\hline Vehicle Type & Car & Truck & Tracked Vehicle \\
\hline Average Accuracy & $90.67 \%$ & $92.86 \%$ & $96.42 \%$ \\
\hline
\end{tabular}


The proposed method realizes the counting and moving direction estimation issue through the proposed algorithm with only $4 \mathrm{~cm}$ aperture array. The method also introduces spatial coherence for wind noise suppression and IAR to overcome the interference from unrelated targets. In the situation of different types of closely spaced vehicles, however, the method failed because the acoustic signals are dominated by very noisy vehicles. Then, the problem of separating signals in multiple target scenarios needs to be solved, which will be considered in future work.

\section{Conclusions}

In this paper, we proposed a SAMA-based single node acoustic monitoring system with only $4 \mathrm{~cm}$ aperture. The proposed method includes vehicle counting and motion direction estimation. The method obtains the required intelligence by analysing the varying trend of a moving vehicle's angles within the vicinity of the CPA. Spatial coherence was assessed to select frequency bands for wind noise suppression and DOA estimation. We applied our proposed system to four different experimental environments, and assessed the accuracy of vehicle counting and motion direction estimation. The experimental results in harsh conditions confirmed the availability of our proposed UGS monitoring system.

Acknowledgments: This work was supported by Sci-tech Innovation Fund of China under Grant No. Y64GYA1001, National Natural Science Foundation of China under Grant No. 14ZR1447200 and National Defense Foundation of China under Grant No. 9140C1801021xxx001. The authors would like to thank the associate editor and anonymous reviewers for their valuable comments and suggestions to improve this paper.

Author Contributions: Xingshui Zu, Feng Guo, and Xin Zhang have contributed to the scientific part of this work. All the authors have contributed to the writing of this article.

Conflicts of Interest: The authors declare no conflict of interest.

\section{References}

1. Toyoda, T.; Ono, N.; Miyabe, S.; Yamada, T.; Makino, S. Vehicle Counting and Lane Estimation with Ad-hoc Microphone Array in Real Road Environments. In Proceedings of the 2016 International Workshop on Nonlinear Circuits, Communications and Signal Processing, Honolulu, HI, USA, 6-9 March 2016; pp. 622-625.

2. Severdaks, A.; Liepins, M. Vehicle counting and motion direction detection using microphone array. Elektron. Elektrotech. 2013, 19, 89-92.

3. Toyoda, T.; Ono, N.; Miyabe, S.; Yamada, T.; Makino, S. Traffic monitoring with ad-hoc microphone array. In Proceedings of the 2014 14th International Workshop on Acoustic Signal Enhancement (IWAENC), Riviera, France, 8-11 September 2014; pp. 318-322.

4. Nesenbergs, K.; Selavo, L. A distributed data processing architecture for real time intelligent transport systems. In Proceedings of the 2013 Second International Conference on Informatics \& Applications (ICIA), Lodz, Poland, 23-25 September 2013.

5. Fernández-Lozano, J.; Martín-Guzmán, M.; Martín-Ávila, J.; García-Cerezo, A. A wireless sensor network for urban traffic characterization and trend monitoring. Sensors 2015, 15, 26143-26169.

6. Heberley, J.R.; Hohil, M.M.; Chang, J.W.; Rotolo, A.G. Method for Detecting Extended Range Motion and Counting Moving Objects Using an Acoustics Microphone Array. U.S. Patent 6,914,854, 5 July 2005.

7. Marszałek, Z.; Sroka, R.; Stencel, M. A new method of inductive sensor impedance measurement applied to the identification of vehicle parameters. Metrol. Meas. Syst. 2011, 18, 69-76.

8. Minge, E.; Kotzenmacher, J.; Peterson, S. Evaluation of Non-Intrusive Technologies for Traffic Detection; Technical Report; Minnesota Department of Transportation, Research Services Section, St. Paul, MN, USA, 2010.

9. Jeon, S.; Kwon, E.; Jung, I. Traffic measurement on multiple drive lanes with wireless ultrasonic sensors. Sensors 2014, 14, 22891-22906.

10. Huang, J.; Zhou, Q.; Zhang, X.; Song, E.; Li, B.; Yuan, X. Seismic Target Classification Using a Wavelet Packet Manifold in Unattended Ground Sensors Systems. Sensors 2013, 13, 8534-8550. 
11. Pancharatnam, M.; Sonnadara, D. Vehicle counting and classification from a traffic scene. In Proceedings of the 26th National IT Conference, Colombo, Sri Lanka, 20-21 August 2008.

12. Taghvaeeyan, S.; Rajamani, R. Portable roadside sensors for vehicle counting, classification, and speed measurement. IEEE Trans. Intell. Transp. Syst. 2014, 15, 73-83.

13. Hohil, M.E. Vehicle counting and classification algorithms for unattended ground sensors. Proc. SPIE 2003, 5090, 99-110.

14. Li, D.; Wong, K.D.; Hu, Y.H.; Sayeed, A.M. Detection, classification, and tracking of targets. IEEE Signal Process. Mag. 2002, 19, 17-29.

15. Huang, J.; Zhang, X.; Guo, F.; Zhou, Q.; Liu, H.; Li, B. Design of an acoustic target classification system based on small-aperture microphone array. IEEE Trans. Instrum. Meas. 2015, 64, 2035-2043.

16. Arora, A.; Dutta, P.; Bapat, S.; Kulathumani, V.; Zhang, H.; Naik, V.; Mittal, V.; Cao, H.; Demirbas, M.; Gouda, M.; et al. A line in the sand: A wireless sensor network for target detection, classification, and tracking. Comput. Netw. 2004, 46, 605-634.

17. Doser, A.B.; Yee, M.L.; O’Rourke, W.T.; Slinkard, M.E.; Craft, D.C.; Nguyen, H.D. Distributed algorithms for small vehicle detection, classification, and velocity estimation using unattended ground sensors. Proc. SPIE 2005, 5796, 374-385.

18. Bahrampour, S.; Ray, A.; Sarkar, S.; Damarla, T.; Nasrabadi, N.M. Performance comparison of feature extraction algorithms for target detection and classification. Pattern Recognit. Lett. 2013, 34, 2126-2134.

19. Blatt, S.R. Multi-Sensor Target Counting and Localization System. U.S. Patent 6,690,321, 10 February 2004.

20. Barbagli, B.; Bencini, L.; Magrini, I.; Manes, G.; Manes, A. A real-time traffic monitoring based on wireless sensor network technologies. In Proceedings of the 2011 7th International Wireless Communications and Mobile Computing Conference (IWCMC), Istanbul, Turkey, 4-8 July 2011; pp. 820-825.

21. Scharrer, R.; Vorländer, M. Sound field classification in small microphone arrays using spatial coherences. IEEE Trans. Audio Speech Lang. Process. 2013, 21, 1891-1899.

22. Cevher, V.; Chellappa, R.; Mcclellan, J.H. Vehicle Speed Estimation Using Acoustic Wave Patterns. IEEE Trans. Signal Process. 2009, 57, 30-47.

23. Zhang, X.; Huang, J.C.; Song, E.L.; Liu, H.W.; Li, B.Q.; Yuan, X.B. Design of Small MEMS Microphone Array Systems for Direction Finding of Outdoors Moving Vehicles. Sensors 2014, 14, 4384-4398.

24. Wilson, D.K.; Greenfield, R.J.; White, M.J. Spatial structure of low-frequency wind noise. J. Acoust. Soc. Am. 2007, 122, 223-228.

25. Carter, G.C. Coherence and time delay estimation. Proc. IEEE 1987, 75, 236-255.

26. Beaufort Scale. Available online: http://en.wikipedia.org/wiki/Beaufort_scale (accessed on 5 August 2016).

27. Lelong, J. Vehicle noise emission: Evaluation of tyre/road and motor-noise contributions. In Proceedings of the Internoise 99-The 1999 International Congress on Noise Control Engineering, Fort Lauderdale, FL, USA, 6-8 December 1999; Volume 1, p. 203.

28. Guarnaccia, C. Advanced tools for traffic noise modelling and prediction. WSEAS Trans. Syst. 2013, $12,121-130$.

(C) 2017 by the authors. Licensee MDPI, Basel, Switzerland. This article is an open access article distributed under the terms and conditions of the Creative Commons Attribution (CC BY) license (http:/ / creativecommons.org/licenses/by/4.0/). 